\title{
Intervención Contra la Caries Dental a Partir de Acciones de Protección Específica en un Grupo de Escolares de Seis a Once Años de la Alcaldía Iztapalapa de la Ciudad de México
}

\author{
Adriano Anaya María del Pilar (PhD), \\ Caudillo Joya Tomás (PhD) \\ FES-Zaragoza UNAM \\ Caudillo Adriano Pilar Alejandra (MA) \\ Práctica Privada \\ Murrieta Pruneda José Francisco (PhD) \\ FES-Zaragoza UNAM
}

\section{Doi:10.19044/esj.2021.v17n37p233}

Submitted: 04 January 2021

Accepted: 08 October 2021

Published: 31 October 2021
Copyright 2021 Author(s)

Under Creative Commons BY-NC-ND 4.0 OPEN ACCESS

Cite As:

N’Guessan A.H., Gogoue D.O., Anougba B.D., Dembélé I. \& Allou K. (2021). Evaluation de Différents Types de Substrats sur Le Développement des Plantules de Palmiers À Huile (Elaeis Guineensis Jacq.) en Côte d'Ivoire. European Scientific Journal, ESJ, 17(37), 233. https://doi.org/10.19044/esj.2021.v17n37p233

\section{Resumen}

Objetivo: Implementar un proyecto de intervención contra la caries dental a partir de acciones de protección específica de la salud bucal con el propósito de reducir la incidencia de caries dental en una población escolar de seis a once años, de la Alcaldía Iztapalapa.

Material y Métodos: Estudio longitudinal descriptivo y de comparación, conformado por un grupo control, con una muestra de 908 escolares de primer año de primaria que representó el $70 \%$ del total de niños de este grado escolar que entregaron su consentimiento firmado previa información a los padres de familia, realizado en 10 escuelas primarias de la Alcaldía Iztapalapa, que se encuentra ubicada en el oriente de la Ciudad de México. Y el grupo testigo conformado por los escolares (3440) que participaron en el diagnóstico base o inicial.

Resultados: Tomando como base los resultados del diagnóstico bucal realizado en 3440 escolares, se llevó a cabo una intervención en el grupo control, con la aplicación de un programa de protección específica a partir de acciones de profilaxis, selladores de fosetas y fisuras y técnica de cepillado, 
así mismo, un seguimiento epidemiológico que inició cuando los escolares se encontraban en primer año y concluyó cuando estos terminaron el cuarto año de primaria, el estudio lo concluyeron 815 escolares. A los escolares que participaron en el grupo testigo no se les realizó ninguna acción odontológica, sus resultados sirvieron sólo de comparación con el propósito de identificar la incidencia de caries dental de los escolares del grupo control una vez concluida la intervención. La información se capturó en el Programa de Cómputo SPSS versión 23.0 en el que se obtuvieron: Estadísticas descriptivas: promedios y DS de caries dental, t de Student, y X2 de Pearson.

Conclusiones: La reducción de la caries dental en el grupo control fue del $70.2 \%$.

Palabras clave: Caries Dental, Protección Específica, Incidencia, Escolares

\title{
Intervention Against Dental Caries Based on Specific Protection Actions in a Group of Schoolchildren Aged Six to Eleven from the Iztapalapa Mayor's Office of Mexico City
}

\author{
Adriano Anaya María del Pilar (PhD), \\ Caudillo Joya Tomás (PhD) \\ FES-Zaragoza UNAM \\ Caudillo Adriano Pilar Alejandra (MA) \\ Práctica Privada \\ Murrieta Pruneda José Francisco (PhD) \\ FES-Zaragoza UNAM
}

Abstract

Objective: To implement an intervention project against dental caries based on specific protection actions for oral health in order to reduce the incidence of dental caries in a school population, aged six to eleven years old, of the Iztapalapa Mayor's Office.

Material and Methods: Longitudinal descriptive and comparison study, made up of a control group, was used in the study. It was carried out on a sample of 908 schoolchildren in the first year of primary school, which represents $70 \%$ of the total number of children of this school grade who gave their signed consent through their parents, The study, however, was carried out in 10 elementary schools of the Iztapalapa Mayor's Office, which is located in the east of Mexico City. The control group is made up of schoolchildren (3440) who participated in the baseline or initial diagnosis.

Results: Based on the results of the oral diagnosis carried out in 3440 schoolchildren, an intervention was carried out in the control group with the 
application of a specific protection program based on prophylactic actions, pit and fissure sealants, and technique of brushing. Likewise, based on an epidemiological follow-up that began when the schoolchildren were in the first year and ended when they finished the fourth year of primary school, the study was completed by 815 schoolchildren. The schoolchildren who participated in the control group did not undergo any dental action, and their results served only as a comparison with the purpose of identifying the incidence of dental caries in the schoolchildren in the control group once the intervention has been concluded. The information was captured using the SPSS Computer Program version 23.0 in which the following were obtained: descriptive statistics such as the means and SD of dental caries, Student's t test, and Pearson's correlation $\mathrm{X} 2$. $70.2 \%$.

Conclusions: The reduction of dental caries in the control group was

Keywords: Dental Caries, Specific Protection, Incidence, School

\section{Introducción}

La colocación de selladores de fosetas y fisuras es un procedimiento seguro, efectivo y económico en la prevención de caries (Villarreal Rojas et al., 2015).

Existen estudios que indican que incluso cuando éstos parecen que se han desprendido totalmente quedan restos de ionómero en el interior de las fisuras lo que actúa aún como una reserva de flúor y de tal forma previene la caries dental (Haznedaroğlu et al., 2016).

Es importante señalar que los sellantes de fosas y fisuras no son sólo para los niños, sino para pacientes de cualquier edad (jóvenes y adultos) así como para personas que sufren pérdida de habilidades motoras debido a la artritis o cualquier otra enfermedad, lo cual significa que tienen que tener un cepillado más prolongado y difícil y en estos casos los sellantes pueden ayudar a proteger los dientes que no están limpiándose constantemente porque el paciente no es capaz de hacer un trabajo minucioso, para poder mantener una boca saludable (Microleakage of New Light-Cure Sealants, 1995; ADA News Releases, 1997). 1999):

Los sellantes tienen 3 efectos preventivos fundamentales (Barrancos,

1) obturan mecánicamente las fosas y fisuras con una resina resistente a los ácidos;

2) al obturar las fosas y fisuras suprimen el hábitat de los streptococcus mutans y otros microorganismos;

3) facilita la limpieza de las fosas y fisuras mediante métodos físicos como el cepillado dental y la masticación. 
Los selladores oclusales, fueron introducidos en 1965 y consisten en aplicar un material en las fosas y fisuras dentales produciendo una barrera mecánica que impide la colonización de bacterias y el paso de los nutrientes necesarios para su reproducción (Pérez, Yamamoto, \& Morales, 2002).

En un estudio con aplicación de selladores en Argentina (Navarro, Ojeda, Herrera \& González, 2004) en 160 escolares los autores manejan una protección del $95 \%$ al 100\% contra la caries dental.

En la Provincia de ciudad real en España (Navarro Montes et al., 2004). se evaluó la aplicación de selladores en un programa público de salud bucodental en población infantil obteniendo un éxito del $88.4 \%$ en la zona urbana.

En Ortiz, B., Sánchez, B. y Delgado, E. (2006), después de 18 meses de iniciado un estudio con selladores en población escolar se encontró una menor prevalencia de caries dental en la evaluación inicial en comparación con la evaluación 18 meses después (84,8\% versus 86,6\% respectivamente), la diferencia entre ambas evaluaciones no resultó estadísticamente significativa $(p=0,687)$. De acuerdo con estos resultados, sólo 2 niños $(1,8 \%)$ desarrollaron caries dental durante el tiempo de intervención evaluado.

En México (Adriano \& Caudillo, 2012), específicamente en la Alcaldía Milpa Alta se aplicó del 2006 al 2012 un proyecto similar logrando una reducción de la caries dental del $79 \%$ en la población escolar demostrando las bondades que tiene la protección específica para el control de la caries dental.

Los selladores de fosetas y fisuras han demostrado su eficacia en el control de la caries dental, de ahí la importancia de este estudio que tuvo el objetivo de reducir la incidencia de caries dental en una población escolar.

\section{Material y Métodos}

Este Proyecto forma parte de la Línea de Investigación Salud Pública en Estomatología con número de registro LI-FESZ-341110 de la Facultad de Estudios Superiores Zaragoza UNAM, y fue evaluado en su metodología y aspectos éticos por el Comité Académico de la Carrera de Cirujano Dentista de ésta Facultad.

El estudio fue longitudinal, de intervención y comparativo, con una duración de cuatro años. Se desarrolló en 10 escuelas primarias en la Alcaldía Iztapalapa, al oriente de la Ciudad de México se ubican en zonas con alto y muy alto nivel de marginación, tomando en cuenta los indicadores del INEGI (Consejo de evaluación del desarrollo social del Distrito Federal. (2011). lo que trae como consecuencia su descuido bucal teniendo esta población otras prioridades como son la alimentación, el transporte, la vivienda entre otros.

Este estudio lo conformaron dos grupos: el grupo control conformado por una muestra de 915 escolares que iniciaron el primer año de primaria y 
que entregaron su consentimiento firmado por el padre de familia o tutor y concluyó cuando estos escolares terminaron el cuarto año.

El grupo testigo integrado por 3440 alumnos de las escuelas primarias participantes, como parte del diagnóstico base o inicial y no se les realizó ninguna acción odontológica sus resultados sirvieron de comparación del grupo control al finalizar la intervención.

La exploración bucal la realizaron cinco pasantes de la carrera de Cirujano Dentista y dos profesores responsables del estudio entrenados y calibrados en el conocimiento y aplicación de los códigos y criterios del indicador CPOD Con el propósito de que la concordancia no se atribuyera al azar, se aplicó el coeficiente de KAPPA, intra e interexaminadores que permite medir las variaciones de las observaciones hechas por los revisores $(K=0.85$; IC $95 \%)$.

Se inicia la intervención en el grupo control, implementando un Programa de Protección Específica (Caudillo, Adriano \& Caudillo, 2020). Vista como el conjunto de acciones odontológicas que se aplican a la población con el propósito de protegerla contra los problemas bucales. Su Objetivo en este proyecto es la reducción en la incidencia de caries dental de los escolares participantes.

Profilaxis. Se realizó cada seis meses a los escolares que participaron en el grupo control.

Aplicación de selladores de fosetas y fisuras en dentición permanente, se sellaban a todos los premolares y molares sanos conforme iban erupcionando, aquellos selladores que se desplazaban total o parcialmente de los dientes se volvían a colocar.

Técnica de cepillado a nivel individual. Se enseñó a cepillarse a todos los escolares del grupo control. Así mismo a los sus padres o tutores con la técnica de Stillman modificada, que acudían cuando se les citaba. A estos escolares se les regaló cepillos dentales para él y su familia cada seis meses.

El seguimiento epidemiológico durante los cuatro años fue realizado con el indicador epidemiológico CPOD, la información se concentró en formatos donde se registró nombre de la escuela, grupo, el nombre, edad y sexo del escolar y la situación de cada uno de los dientes revisados colocando los siguientes códigos: 1. Cariado, 2. Perdido, 3. Obturado, 4. Sellado, 5. Sano, 6. No erupcionado. Al finalizar el estudio se obtuvo el CPOS con el propósito de identificar las superficies dentales que presentaron más caries dental, utilizando los códigos y criterios recomendados por la OMS (Encuestas de salud bucodental \& Métodos básicos, 1999).

Se capacitó a los maestros de las escuelas primarias participantes sobre la técnica de cepillado de Stillman modificada, con el propósito de supervisar a los escolares en el cepillado de sus dientes después de ingerir su refrigerio y 
con ello garantizar que por lo menos una vez al día realizaban esta actividad de manera adecuada.

\section{Diseño Estadístico del studio}

La información se capturó en el Programa de Cómputo SPSS versión 23.0 en el que se obtuvieron: Estadísticas descriptivas: promedios y DS de caries dental, t de Student, y X2 de Pearson. Se consideraron los resultados estadísticamente significativos cuando el valor p sea menor a 0.05.

Finalmente se llevó a cabo la discusión y las conclusiones del proyecto de intervención

\section{Conflicto de intereses}

No existe conflicto de intereses.

\section{Resultados}

Tanto los escolares del grupo control cono del testigo, pertenecieron a las mismas primarias,

La Alcaldía Iztapalapa otorgó el permiso para realizar el estudio en diez, escuelas primarias públicas, distribuidas en tres regiones educativas, los resultados del Diagnóstico base (grupo testigo), realizado previo del inicio del proyecto de intervención, indica que los promedios generales de caries dental por cada una de las primarias van de 3.0 a 3.3 dientes permanentes con historia de la enfermedad. Se puede observar como los promedios de caries dental van aumentando conforme a la edad (Tabla 1).

\begin{tabular}{|c|c|c|c|c|c|c|c|c|c|c|}
\hline \multicolumn{10}{|c|}{ Tabla 1. Distribución del promedio de caries dental por primaria y edad, del grupo testigo } \\
\hline & \multicolumn{9}{|c|}{ Edad de los escolares } & \\
\hline Primaria & No. & 6 & 7 & 8 & 9 & 10 & 11 & 12 & CPOD & $\begin{array}{c}\text { Regiones } \\
\text { educativas }\end{array}$ \\
\hline $\begin{array}{c}\text { 1.- José } \\
\text { Palomares }\end{array}$ & 470 & 0.8 & 1.7 & 2.4 & 3.0 & 3.7 & 4.5 & 5.4 & $3.1 \pm 2.1$ & Centro \\
\hline $\begin{array}{c}\text { 2.- Antonio } \\
\text { Castro Leal }\end{array}$ & 192 & 1.0 & 2.1 & 2.6 & 3.5 & 4.3 & 5.2 & NO & $3.1 \pm 1.8$ & Juárez \\
\hline $\begin{array}{c}\text { 3.- Ignacio } \\
\text { Chávez }\end{array}$ & 408 & 1.2 & 1.7 & 2.4 & 3 & 3.7 & 4.4 & 5 & $3.1 \pm 2.0$ & Juárez \\
\hline $\begin{array}{c}\text { 4.- Julio } \\
\text { Cortázar }\end{array}$ & 354 & 0.9 & 2 & 2.4 & 3.5 & 4.3 & 4.8 & 5.0 & $3.1 \pm 2.1$ & Juárez \\
\hline $\begin{array}{c}\text { 5.- Miguel de } \\
\text { Cervantes }\end{array}$ & 364 & 1.2 & 1.6 & 2.9 & 3.6 & 3.9 & 4.5 & 4.7 & $3.2 \pm 2.0$ & Centro \\
\hline $\begin{array}{c}\text { 6.- Emperador } \\
\text { Izcoatl }\end{array}$ & 511 & 0.8 & 1.2 & 2.3 & 3.2 & 4.1 & 4.3 & 5.0 & $3.0 \pm 2.6$ & Juárez \\
\hline $\begin{array}{c}\text { 7.- Mitla } \\
\text { I.- Juan de Mata }\end{array}$ & 197 & 1.1 & 1.9 & 2.8 & 3 & 3.5 & 4.1 & 5.1 & $3.1 \pm 2.3$ & San Lorenzo \\
\hline $\begin{array}{c}\text { 9.- Miguel } \\
\text { Sánchez }\end{array}$ & 205 & 1.1 & 1.4 & 2.3 & 3.2 & 3.9 & 4.4 & 5.0 & $3.0 \pm 2.0$ & San Lorenzo \\
\hline
\end{tabular}




\begin{tabular}{|c|c|c|c|c|c|c|c|c|c|c|}
\hline $\begin{array}{c}\text { 10.- Fray } \\
\text { Bartolomé }\end{array}$ & 407 & 0.6 & 1.5 & 2.6 & 3.2 & 4 & 5 & 6.3 & $3.3 \pm 2.4$ & San Lorenzo \\
\hline Total & 3440 & 1.0 & 1.7 & 2.6 & 3.2 & 3.7 & 4.4 & 5.0 & $3.0 \pm 2.2$ & \\
\hline \multicolumn{10}{|c|}{ ANOVA $=0.000$} \\
\hline
\end{tabular}

\section{Primer año de la intervención (grupo control)}

El estudio se inicia con 908 escolares de primer año de primaria que entregaron su consentimiento informado previa información a los padres de familia de los objetivos del mismo. Después de haber realizado las acciones odontológicas a los escolares, al finalizar el ciclo escolar, los promedios de caries dental por cada una de las primarias van de 0.1 a 0.5 dientes con historia de la enfermedad. Al asociar los promedios de caries dental por cada una de las escuelas se encontró significancia estadística $(\mathrm{p}=0.00)$. El promedio general fue de 0.3 dientes cariados (Tabla 2).

Tabla 2. Promedios de caries dental al finalizar el primer año del estudio por primaria y número de escolares participantes en el grupo control

\begin{tabular}{|c|c|c|c|}
\hline Escuela & No. & $\%$ & CPOD \\
\hline 1.- José Palomares & 117 & 12.9 & $0.5 \pm 1.0$ \\
\hline 2.- Ignacio Chávez Matutino & 75 & 8.3 & $0.2 \pm 0.4$ \\
\hline 3.- Fray Bartolomé & 78 & 8.6 & $0.2 \pm 0.5$ \\
\hline 4.- Castro Leal Matutino & 76 & 8.4 & $0.2 \pm 0.4$ \\
\hline 5.- Julio Cortázar Matutino & 91 & 10.0 & $0.1 \pm 0.3$ \\
\hline 6.- Mitla Matutino & 73 & 8.0 & $0.1 \pm 0.3$ \\
\hline 7.- Miguel de Cervantes & 127 & 14.0 & $0.5 \pm 0.9$ \\
\hline 8.- Juan de Mata & 52 & 5.7 & $0.2 \pm 0.6$ \\
\hline 9.- Emperador Azteca & 97 & 10.7 & $0.5 \pm 1.0$ \\
\hline 10.- Miguel Sánchez & 122 & 13.4 & $0.2 \pm 0.6$ \\
\hline Total & 908 & 100 & $0.3 \pm 0.7$ \\
\hline
\end{tabular}

Del total de escolares (908), del grupo control, el 85.2\% correspondió a la edad de seis años, encontrando sólo el 3.7\% de la muestra a los ocho (Tabla 3).

\begin{tabular}{|c|c|c|c|}
\hline & \multicolumn{3}{|c|}{$\begin{array}{l}\text { Tabla 3. Distribución de los escolares participantes del } \\
\text { grupo control por edad }\end{array}$} \\
\hline Edad & No. & $\%$ & CPOD \\
\hline 6 & 774 & 85.2 & 0.2 \\
\hline 7 & 100 & 11.0 & 0.4 \\
\hline 8 & 34 & 3.7 & 0.5 \\
\hline Total & 908 & 100 & 0.3 \\
\hline
\end{tabular}

Con respecto al sexo del grupo control el 52\% corresponde al femenino y el $48 \%$ al masculino. Los promedios de caries dental fueron similares en ambos (Tabla 4). 


\begin{tabular}{|c|c|c|c|}
\hline \multicolumn{4}{|c|}{ Tabla 4. Promedios de caries dental de los escolares del } \\
grupo control por sexo \\
\hline Sexo & No. & $\%$ & CPOD \\
\hline Masculino & 438 & 48.0 & 0.3 \\
\hline Femenino & 470 & 52.0 & 0.3 \\
\hline Total & 908 & 100 & 0.3 \\
\hline
\end{tabular}

En el primer año del estudio, a la revisión encontramos que de los primeros molares permanentes, el superior derecho fue el menos erupcionado (50.6\%), los más cariados fueron los inferiores izquierdos (9.7\%) y derecho (9.8\%), en el primer año de estudio no se encontraron dientes obturados (cuadro 4).

Se sellaron sanos 1561 molares y se encontraban cariados al momento de la revisión 263 (Tabla 5).

Tabla 5. Situación de los dientes permanentes de los escolares en el primer año de la intervención

\begin{tabular}{|c|c|c|c|c|c|c|c|c|c|c|}
\hline & \multicolumn{2}{|c|}{ Sanos } & \multicolumn{2}{c|}{$\begin{array}{c}\text { Sellados } \\
\text { sanos }\end{array}$} & \multicolumn{2}{c|}{$\begin{array}{c}\text { No } \\
\text { erupcionados }\end{array}$} & \multicolumn{2}{c|}{ Cariados } & \multicolumn{2}{c|}{ Total } \\
\hline Dientes & No. & $\%$ & No. & $\%$ & No. & $\%$ & No & $\%$ & No. & $\%$ \\
\hline 16 & - & - & 409 & 45.0 & 459 & 50.6 & 40 & 4.4 & 908 & 100 \\
\hline 12 & 116 & 12.8 & - & - & 792 & 87.2 & - & - & 908 & 100 \\
\hline 11 & 297 & 32.7 & - & - & 611 & 67.3 & - & - & 908 & 100 \\
\hline 21 & 301 & 33.1 & - & - & 607 & 66.9 & - & - & 908 & 100 \\
\hline 22 & 141 & 15.5 & - & - & 767 & 84.5 & - & - & 908 & 100 \\
\hline 26 & - & - & 405 & 44.6 & 457 & 50.3 & 46 & 5.1 & 908 & 100 \\
\hline 36 & - & - & 369 & 40.6 & 451 & 49.7 & 88 & 9.7 & 908 & 100 \\
\hline 32 & 234 & 30.0 & - & - & 552 & 70.0 & - & - & 908 & 100 \\
\hline 31 & 503 & 44.6 & - & - & 405 & 44.6 & - & - & 908 & 100 \\
\hline 41 & 504 & 55.5 & - & - & 404 & 44.5 & - & - & 908 & 100 \\
\hline 42 & 240 & 31.0 & - & - & 546 & 69.0 & - & - & 908 & 100 \\
\hline 46 & - & - & 378 & 41.6 & 441 & 48.6 & 89 & 9.8 & 908 & 100 \\
\hline & 2336 & & 1561 & & 6492 & & 263 & & & \\
\hline
\end{tabular}

Al finalizar el segundo y tercer año de la intervención los promedios de caries dental de los escolares del grupo control (Tabla 6) fue de 0.4 y 0.6 dientes con historia de la enfermedad respectivamente (Gráfico 1). 


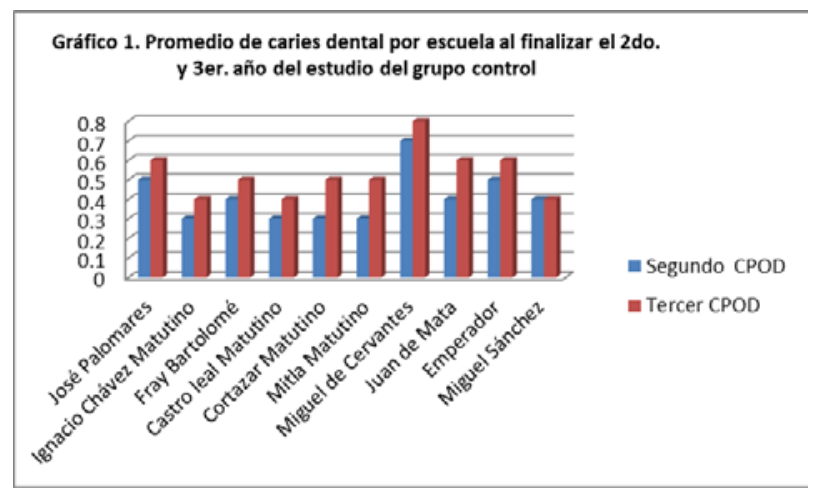

A la revisión de los escolares del grupo control, con respecto a los primeros molares sanos se sellaron 2173 aproximadamente un 60\% de ellos. En este segundo año de la intervención se dieron de baja 26 escolares por cambio de domicilio.

Al finalizar el tercer año de la intervención, de los 908 estudiantes que iniciaron la intervención se habían dado de baja 54 más de ellos, quedando el grupo control con 854 estudiantes, El 71\% de los molares permanentes se encontraron sellados (2585), y el 6.2\% de los primeros premolares (226).

\section{Cuarto año de la intervención}

Al finalizar el cuarto año del estudio, el promedio general fue de 0.8 dientes con historia de caries dental por cada escolar. El promedio más elevado lo encontramos en la escuela Mitla turno matutino y Juan de Mata. No se encontró diferencias estadísticas entre la caries dental y su distribución por cada una de las escuelas ( $\mathrm{p}=0.302)$ (Gráfico 2).

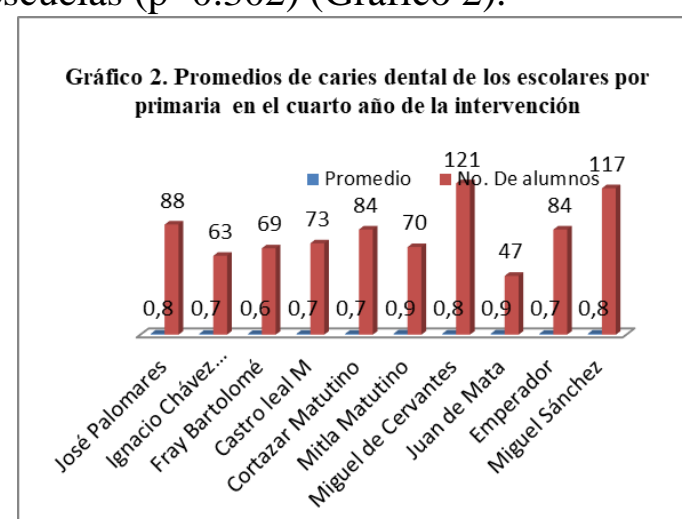

Al finalizar el cuarto año de la intervención los promedios de caries dental con respecto a la edad van de 0.7 a los nueve años a 0.9 dientes con historia de esta enfermedad a los diez y once años, no encontrando diferencias estadísticas entre ambas variables ( $\mathrm{p}=0.074)$ (Gráfico 3). 


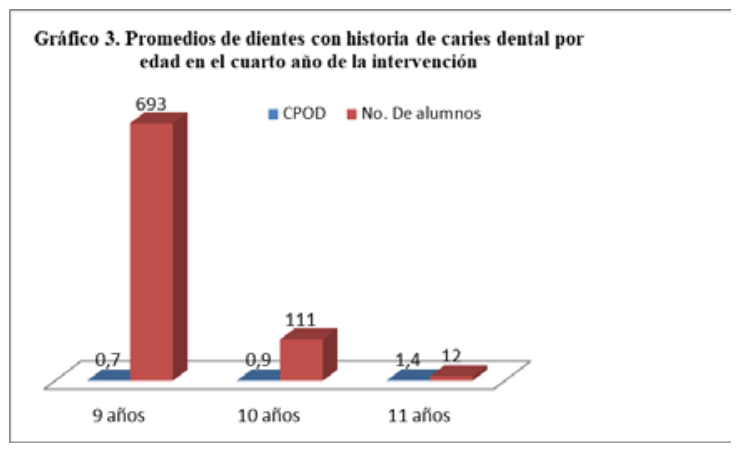

Al finalizar el cuarto año de la intervención 92, escolares del grupo control se dieron de baja del estudio por cambio de la primaria.

El primer molar permanente más cariado fue inferior izquierdo (36). El menos el superior derecho (16), sólo se encontró un diente cariado en los anteriores, el central inferior izquierdo (Tabla 6).

\begin{tabular}{|c|c|c|c|c|c|c|c|c|c|c|}
\hline \multirow[b]{2}{*}{ Dientes } & \multicolumn{2}{|c|}{ Sanos } & \multicolumn{2}{|c|}{ Sellados sanos } & \multicolumn{2}{|c|}{$\begin{array}{l}\text { No } \\
\text { erupcionados }\end{array}$} & \multicolumn{2}{|c|}{ Cariados } & \multicolumn{2}{|c|}{ Obturados } \\
\hline & No. & $\%$ & No. & $\%$ & No. & $\%$ & No. & $\%$ & No. & $\%$ \\
\hline 17 & - & - & 15 & 1.8 & 800 & 98.2 & - & - & - & - \\
\hline 16 & - & - & 735 & 90.2 & 3 & 0.4 & 66 & 8.1 & 11 & 1.3 \\
\hline 15 & - & - & 492 & 60.4 & 323 & 39.6 & - & - & - & - \\
\hline 14 & - & - & 519 & 63.7 & 296 & 36.3 & - & - & - & - \\
\hline 13 & 9 & 1.1 & - & - & 806 & 98.9 & - & - & - & - \\
\hline 12 & 815 & 100 & & & - & - & - & - & - & - \\
\hline 11 & 813 & 99,8 & - & - & 2 & 0,2 & - & - & - & - \\
\hline 21 & 810 & 99.4 & - & - & 5 & 0.6 & - & - & - & - \\
\hline 22 & 812 & 99.6 & - & - & 3 & 0.4 & - & - & - & - \\
\hline 23 & 12 & 1.5 & - & - & 803 & 98.5 & - & - & - & - \\
\hline 24 & - & - & 337 & 41.3 & 478 & 58.7 & - & - & - & - \\
\hline 25 & - & - & 150 & 18.4 & 665 & 81.6 & - & - & - & - \\
\hline 26 & - & - & 701 & 86.0 & 18 & 2.2 & 77 & 9.4 & 19 & 2.3 \\
\hline 27 & - & - & 34 & 3.7 & 781 & 86.0 & - & - & & - \\
\hline 37 & - & - & 37 & 4.5 & 778 & 95.5 & - & - & - & - \\
\hline 36 & - & - & 613 & 75.2 & 11 & 1.3 & 129 & 15.8 & 62 & 7.6 \\
\hline 35 & - & - & 129 & 15.8 & 686 & 84.2 & - & - & - & - \\
\hline 34 & - & - & 320 & 39.3 & 495 & 60.7 & - & - & - & - \\
\hline 33 & 15 & 1.8 & - & - & 800 & 98.2 & - & - & - & - \\
\hline 32 & 812 & 99.6 & - & - & 3 & 0.4 & - & - & - & - \\
\hline 31 & 814 & 99.9 & - & - & - & - & 1 & 0.1 & - & - \\
\hline 41 & 815 & 100 & - & - & - & - & - & - & - & - \\
\hline 42 & 815 & 100 & - & - & - & - & - & - & - & - \\
\hline 43 & 13 & 1.6 & - & - & 802 & 98.4 & - & - & - & - \\
\hline 44 & - & - & 345 & 42.3 & 470 & 57.7 & - & - & - & - \\
\hline
\end{tabular}




\begin{tabular}{|l|l|l|l|l|l|l|l|l|l|l|}
\hline 45 & & & 167 & 20.5 & 648 & 79.5 & - & - & - & - \\
\hline 46 & - & - & 623 & 76.4 & 7 & 0.9 & 121 & 14.8 & 64 & 7.9 \\
\hline 47 & - & - & 11 & 1.3 & 804 & 98.7 & - & - & - & - \\
\hline Total & 6555 & & 5228 & & 10487 & & 394 & & 156 & \\
\hline
\end{tabular}

Terminaron el cuarto año de la intervención, libres de caries dental 501 escolares y 315 con historia de caries dental.

Al finalizar el cuarto año de la intervención el $48 \%$ de los escolares fueron del sexo masculino con un promedio de 0.8 dientes cariados y el 52\% del sexo femenino con 0.7 dientes cariados no se encontró diferencias entre ambos ( $\mathrm{p}=0.837)$.

$\mathrm{Al}$ asociar los promedios de caries dental del grupo control y testigo encontramos que al finalizar el primer año de la intervención la incidencia de caries dental es de medio diente más en los escolares del grupo testigo con relación al grupo intervención (control).

En el segundo año la incidencia es de un diente cariado más en el grupo testigo con relación al control.

En el tercer año es de 1.6 y en el cuarto año la incidencia es de dos dientes cariados más en el grupo testigo o diagnóstico base que en el grupo intervención o control (Gráfico 4).

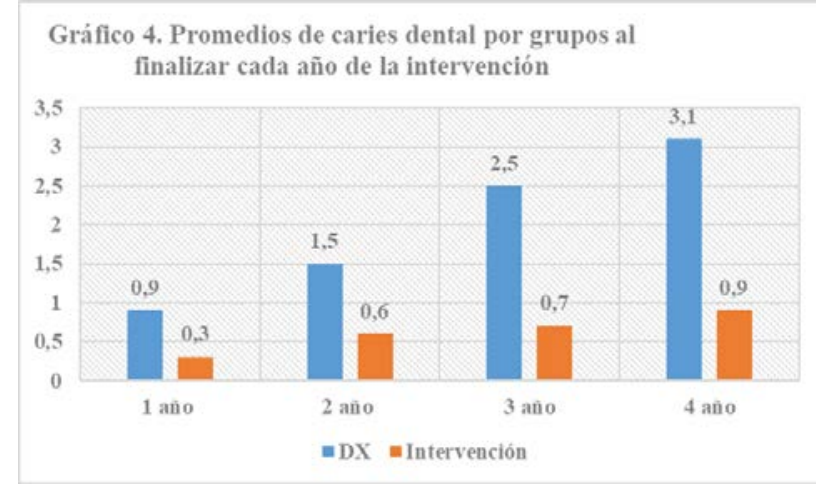

Con respecto a la edad de los escolares en los dos grupos, a los seis y siete años la incidencia de caries dental es de un diente cariado más en el grupo testigo o diagnóstico base. A los ochos años es de dos dientes cariados, a los nueve aumentó a casi 2.5, a los diez años de tres dientes y a los once años de 3.7 dientes cariados más en el grupo testigo o del diagnóstico base que en el intervención o grupo control (Gráfico 5). 


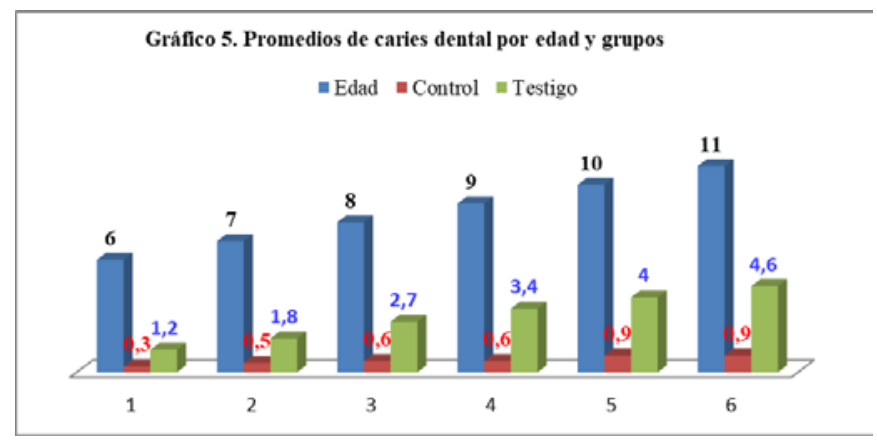

En el cuarto año de la intervención encontramos 12361 dientes erupcionados, lo que nos hace tener 61801 superficies dentales de las cuales el $0.6 \%$ se encontraron con caries dental y solamente el $0.2 \%$ obturadas.

El promedio de superficies con historia de caries dental por escolar fue de 0.6 superficies dañadas. Esto quiere decir que de cada dos niños vamos a encontrar 1.2 superficies dentales afectadas (Tabla 7).

\begin{tabular}{|c|c|c|c|c|c|c|c|}
\hline \multicolumn{7}{|c|}{ Tabla 7. Total de superficies cariadas } \\
\hline $\begin{array}{c}\text { Con 1 superficie } \\
\text { cariada }\end{array}$ & $\begin{array}{c}\text { Con 2 superficies } \\
\text { cariadas }\end{array}$ & \multicolumn{2}{c|}{$\begin{array}{c}\text { Con 3 superficies } \\
\text { cariadas }\end{array}$} & $\begin{array}{c}\text { Con 5 superficies } \\
\text { cariadas }\end{array}$ \\
\hline CO & 231 & COV & 65 & COMV & 24 & COMDLV & 6 \\
\hline CP & 1 & COM & 8 & COMD & 52 & & \\
\hline CV & 5 & & & COMP & 3 & & \\
\hline & & & & CODV & 2 & & \\
\hline Total & 237 & Total & 73 & Total & 81 & Total & 6 \\
\hline \multicolumn{78}{|c|}{ Total 397 superficies cariadas } \\
\hline
\end{tabular}

Se encontraron también en la cuarta revisión 154 superficies obturadas, 82 de éstas corresponden a la oclusal, 30 a la ocluso mesial y 21 a la ocluso mesio distal (Tabla 8).

\begin{tabular}{|c|c|c|c|c|c|c|c|c|c|}
\hline \multicolumn{10}{|c|}{ Tabla 8. Total de superficies obturadas } \\
\hline \multicolumn{2}{|c|}{$\begin{array}{c}\text { Con } 1 \\
\text { superficie } \\
\text { obturada }\end{array}$} & \multicolumn{2}{|c|}{$\begin{array}{c}\text { Con } 2 \\
\text { superficies } \\
\text { obturadas }\end{array}$} & \multicolumn{2}{|c|}{$\begin{array}{c}\text { Con } 3 \\
\text { superficies } \\
\text { obturadas }\end{array}$} & \multicolumn{2}{|c|}{$\begin{array}{c}\text { Con } 4 \\
\text { superficies } \\
\text { obturadas }\end{array}$} & \multicolumn{2}{|c|}{$\begin{array}{c}\text { Con } 5 \text { superficies } \\
\text { obturadas }\end{array}$} \\
\hline $\mathrm{OO}$ & 82 & OOM & 30 & OOMD & 21 & OOMDV & 1 & OOMDVP & 3 \\
\hline $\mathrm{OV}$ & 4 & OOP & 5 & & & & & OOMDVL & 3 \\
\hline & & OOV & 4 & & & & & & \\
\hline & & OOL & 1 & & & & & & \\
\hline Total & 86 & Total & 40 & Total & 21 & Total & 1 & Total & 6 \\
\hline
\end{tabular}

\section{Discusión}

Con el objetivo de reducir la incidencia de caries dental en la población escolar de seis a once años de diez escuelas primarias públicas en la Alcaldía Iztapalapa en un 50\%, se implementó un proyecto de intervención, en el grupo 
control a partir de un programa de protección específica, en 908 escolares cuyos padres de familia dieron su consentimiento firmado.

El total de escolares que concluyeron el cuarto año de la intervención fueron 815.

El grupo testigo estuvo conformado por 3440 escolares a los que no se les realizó ninguna acción odontológica y correspondieron al diagnóstico inicial o base.

Las acciones bucales realizadas a los escolares del grupo control, no tuvieron ningún costo para los padres de familia y son consideras de alta cobertura y de bajo costo, y nos permiten controlar la incidencia de los principales problemas bucales como son la caries dental y las periodontopatías, que son la primera causa de dolor y abatir la visita al dentista por urgencia.

El promedio de caries dental en el cuarto año de la intervención del grupo control fue 0.8 dientes con historia de esta enfermedad, mientras que en el grupo testigo fue de 3.1 dientes afectados encontrando 2.3 dientes con más caries en este.

El 61.3\% (500), de los escolares del grupo control terminaron sanos el cuarto año de la intervención. Mientras que 38.7\% (315) de los escolares presentaron caries dental.

La reducción de la caries dental en los cuatro años de duración del estudio fue del $70.2 \%$ por debajo de otros estudios como los realizados en Argentina, (95\%), España, (88.4\%), Perú (84-88\%) y México (79\%), que puede ser resultados que este estudio se llevó a cabo dentro de las escuelas primarias y nos teníamos que adaptar a los contextos de cada una de ellas..

\section{Conclusiones}

Las acciones de protección específica domostraron ser eficaces en la disminución de la incidencia de caries dental en la población escolar de esta alcaldía.

La disminución de la incidencia de caries dental en los escolares del grupo control fue de $70.2 \%$, con un promedio de caries dental de 0.8 dientes con historia de la enfermedad.

\section{References:}

1. ADA News Releases (2020). Disponible en: http://www.ada.org/publiv/media/newsrel/archive.html\#1997. (Consultado 8 de Septiembre).

2. Adriano, P. \& Caudillo, T. (2012). Reducción de la caries dental en un $79 \%$ en escolares de la delegación Milpa Alta. Ciudad de México. Rev. Odontología Actual; 10(122): 4-13. 
3. Barrancos, J. (1999). Operatoria Dental. 3era. ed. Ed. Médica Panamericana; pp. 454-470.

4. Caudillo, T., Adriano, MP. \& Caudillo, PA. (2020). Modelo Estomatológico de Práctica Profesional Integral. Facultad de Estudios Superiores Zaragoza UNAM México.

5. Consejo de evaluación del desarrollo social del Distrito Federal (2011). Índice de desarrollo social de las unidades territoriales del Distrito Federal, delegación, colonia y manzana. México DF. Disponible: http://www.evalua.df.gob.mx/files/indice/ind_inf.pdf. (Consultado 15 de septiembre 2020).

6. Encuestas de salud bucodental \& Métodos básicos (1999). Organización Mundial de la Salud. 13-15.

7. Haznedaroğlu, E., Güner, Ş., Duman, C., Menteş, \& Ali, A. (2016). 48-month randomized controlled trial of caries prevention effect of a one-time application of glass ionomer sealant versus 63 resin sealant. NCBI. 35(3): 532-538. Disponible en:

https://www.ncbi.nlm.nih.gov/pubmed/27086573 (Consultado 24 de marzo 2021).

8. Microleakage of New Light-Cure Sealants (1995). A supplement of Practical periodontics and Aesthetic Dentistry. Supplement $\mathrm{N}^{\circ} 1$.

9. Navarro, MI., Ojeda, L., Herrera, MA., \& González, SA. (2004). Evaluación de la aplicación de selladores en el marco asistencial de un programa público de salud bucodental. Odontoestomatol; 20(1): 3340.

10. Organización Mundial de la Salud (2020). Determinantes sociales de la salud. Disponible en: https://www.who.int/social_determinants/es/. (Consultado 15 de septiembre septiembre).

11. Ortiz, B., Sánchez, B. \& Delgado, E. (2006). Efectividad de una intervención comunitaria en salud oral: Resultados después de 18 meses. Rev. Med. Heredia, Lima. 17 (3), pp.170-176.

12. Pérez, I., Yamamoto, NA. \& Morales, C. (2002). Estudio comparativo de micro filtración de una resina fluida utilizada como sellador de fosetas y fisuras contra un sellador con relleno utilizando una técnica combinada de grabado ácido con micro abrasión. Disponible: http://www.pve.unam.mx/alerta/pdf/comparativo.pdf. (Consultado 8 de septiembre 2020).

13. Villarreal, RA., Guerrero, IJ., Yamamoto, NA. \& Barceló, SF. (2015). Profundidad de curado de selladores de fosetas y fisuras utilizando luz emitida por diodos (LED) a diferentes distancias. Revista odontológica mexicana, 19(2), 76-80. 